\title{
Clinical Challenges and Considerations in Management of Chronic Pain Patients During a COVID-19 Pandemic
}

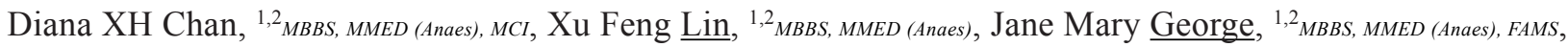

Christopher W Liu, ${ }^{1,2}$ MBBS (Honors), MMED (Anaes)

\begin{abstract}
Since the coronavirus disease 2019 (COVID-19) was deemed a pandemic on 11 March 2020, we have seen exponential increases in the number of cases and deaths worldwide. The rapidly evolving COVID-19 situation requires revisions to clinical practice to defer non-essential clinical services to allocate scarce medical resources to the care of the COVID-19 patient and reduce risk to healthcare workers.

Chronic pain patients require long-term multidisciplinary management even during a pandemic. Fear of abandonment, anxiety and depression may increase during this period of social isolation and aggravate pain conditions. Whilst physical consults for chronic pain patients were reduced, considerations including continuity of support and analgesia, telemedicine, allied health support and prioritising necessary pain services and interventions, were also taken to ensure biopsychosocial care for them.

Chronic pain patients are mostly elderly with multiple comorbidities, and are more susceptible to morbidity and mortality from COVID-19. It is imperative to review pain management practices during the COVID-19 era with respect to infection control measures, re-allocation of healthcare resources, community collaborations, and analgesic use and pain interventions. The chronic pain patient faces a potential risk of functional and emotional decline during a pandemic, increasing healthcare burden in the long term. Clinical decisions on pain management strategies should be based on balancing the risks and benefits to the individual patient.

In this commentary, we aim to discuss the basis behind some of the decisions and safeguards that were made at our tertiary pain centre over the last 6 months during the COVID-19 outbreak.
\end{abstract}

Ann Acad Med Singap. 2020;49:669-73

\section{Introduction}

In December 2019, a novel coronavirus was identified as the cause of a cluster of severe pneumonia cases in Wuhan, China. Since then, the coronavirus has been named Severe Acute Respiratory Distress Syndrome (SARS) Coronavirus (CoV) 2 (or SARSCoV-2) and the disease caused by the virus has been named coronavirus disease 2019 (or COVID-19). By March 2020, COVID-19 had become a global pandemic of unprecedented scale, affecting $>200$ countries. At the time of writing, COVID-19 has infected more than 10.2 million patients globally and has taken the lives of at least 502,000 people. Singapore itself recorded a total of 43,661 cases and 26 fatalities by 29 June 2020.

Pain physicians in Singapore are mostly anaesthetists who may be deployed at the peak of the COVID-19 pandemic to run intensive care units, community and swab facilities, and deliver anaesthesia for COVID-19 patients who may require emergent surgeries. In the midst of our COVID-19 duties, there is still a need to ensure continued support for our chronic pain patients who may require urgent pain consultations, interventions or medication titration and refills. Here we discuss

\footnotetext{
${ }^{1}$ Division of Anaesthesiology, Department of Pain Medicine, Singapore General Hospital, Singapore

${ }^{2}$ Division of Anaesthesiology, Department of Pain Medicine, Sengkang General Hospital, Singapore

Address for Correspondence: Dr Diana XH Chan, Department ofAnaesthesiology,Academia, Level 5, Singapore General Hospital, Outram Road, Singapore 169608

Email: diana.chan.x.h@singhealth.com.sg
} 
considerations for pain management strategies and analgesic use in the midst of the threat of COVID-19, and measures that have been put in place to continue to provide support to our various pain populations.

\section{Pain Interventions and Corticosteroid Use}

A Joint Statement by American Society of Regional Anaesthesia and Pain Medicine (ASRA) and European Society of Regional Anaesthesia and Pain Therapy (ESRA) recommended that no elective pain procedures, except specific urgent and semi-urgent procedures, should be performed during COVID-19 pandemic. ${ }^{1}$ However, categorising pain procedures as elective, urgent or emergent is subjective in many cases. It is important to note that the pain patients at risk (e.g. elderly, multiple comorbidities) of COVID-19 and its complications are also the same at-risk population who is susceptible to complications of immobility and functional deterioration from severe distressing and debilitating pain such as deep vein thrombosis and pulmonary embolism, urinary tract infections, muscle atrophy and stiffness, bed sores and more infections, major depression and other sequelae. As such, individualised assessments of benefits and risks of pain interventions must be exercised.

Corticosteroids were advocated for the treatment of SARS-CoV as well as MERS-CoV because it was thought that corticosteroids could blunt the host immune response and therefore suppress pulmonary inflammation. ${ }^{2}$ However, studies performed during these outbreaks not only failed to show a mortality benefit from the use of corticosteroids but also demonstrated possible harm. ${ }^{3-7}$ Consequently, the World Health Organization (WHO) recommends against the use of corticosteroids for the treatment of COVID-19 outside of a clinical trial setting. ${ }^{8}$ A statement by the Faculty of the Pain Medicine of Royal College of Anaesthetists also addressed the potential harm of steroid injection to individuals who might be incubating or later develop COVID-19. ${ }^{9}$

Although steroid injections for certain pain conditions are given locoregionally to the affected tissues, there is still systemic uptake of the medication. A recent randomised controlled trial found that the use of particulate steroids in a single short epidural injection led to a significant decrease in morning cortisol levels at 3 months, suggesting that corticosteroid injection can lead to short-term adrenal suppression. ${ }^{10}$ There is also weak evidence suggesting that short term use of oral corticosteroids can result in a significant immunosuppression. ${ }^{11}$ This may have potential implications of delayed COVID-19 presentation and reduced immune response to the virus. Patients should be counselled of these potential implications during informed consent taking should they require steroid injections for pain control. The use of non-particulate steroids (such as dexamethasone) over particulate steroids may also be considered as a risk mitigation strategy if the efficacy of the injection is not compromised. This is based on limited evidence that dexamethasone causes a shorter and unsustained duration of adrenal suppression which is likely to be secondary to its lack of depot effect. ${ }^{10,12}$ In addition, there is also emerging preliminary evidence that dexamethasone use in COVID-19 patients reduced 28-day mortality among those receiving invasive mechanical ventilation or oxygen in an ongoing randomised controlled trial. ${ }^{13}$

It is important to note that steroid usage is only one component of interventional pain management. If pain is severe and function is significantly impaired, advanced pain management techniques and steroidsparing alternatives such as nucleoplasty, annuloplasty, radiofrequency (RF) neurotomy and other neurolytic techniques should be considered whenever possible and in a timely manner. ${ }^{14}$ Withholding pain interventions might potentially lead to significant morbidity, functional deterioration and over-reliance on opioid therapy. As the COVID-19 situation continues to evolve, it is best to make a balanced decision based on the individual patient's needs and medical condition.

\section{Non-Steroidal Anti-Inflammatory Drugs (NSAIDS)}

NSAIDS are commonly used for pain managementeither prescribed by physicians or self-medicated by patients. Use of NSAIDs in the confirmed or suspected COVID-19 patient has been controversial. Given that ibuprofen may upregulate angiotensin-converting enzyme 2 (ACE2) and that SARS-CoV-2 binds to their target cells by binding to ACE2, it has been questioned if ibuprofen use can facilitate COVID-19 infections. ${ }^{15}$ The use of NSAIDs has also been found to be associated with higher rates of complications in patients with community acquired pneumonia. ${ }^{16}$ The National Agency for the Safety of Medicines and Health Products (ANSM) of France thus issued a warning in April 2019 that NSAID use may worsen the severity of existing infections. ${ }^{17}$

Despite these warnings, there is currently no clear evidence linking the use of NSAIDS to poorer outcomes following COVID-19. There are also contrary opinions that anti-inflammatories may help to reduce pulmonary inflammation and several studies are underway to look at whether anti-inflammatories (such as IL-1 
and IL-6 inhibitors) can benefit patients stricken with COVID-19. ${ }^{18}$

In response to this issue, several organisations including the WHO, Food and Drug Administration (FDA) and the Ministry of Health in Singapore $(\mathrm{MOH})$ have recommended that there is no need to avoid NSAIDS. Notably, as compared to corticosteroids, NSAIDS have a short duration of action. Hence, if new evidence emerges, the cessation of NSAIDS will rapidly revert the risk level of patients back to baseline. As such, until further clarity is obtained, NSAIDs can be used as indicated during the COVID-19 pandemic, keeping the duration of therapy to the minimum and using the lowest possible doses.

\section{Opioid Prescription}

Although opioids are commonly prescribed in pain clinics, there is now emerging evidence that opioid therapy may not result in sustained pain relief when used long-term. ${ }^{19}$ Furthermore, it is recognised that chronic opioid therapy may cause immunosuppression, with heterogeneous effects observed in different opioids. ${ }^{20,21}$ In the context of COVID-19, this immunosuppression can affect patients negatively by increasing the risk of infection or reducing their ability to mount an appropriate immune response.

For these reasons, opioid therapy should be used only after an adequate trial of non-opioid therapy. An opioid with minimal immunosuppressive effect should be prescribed if possible, starting at the lowest dose possible and titrated according to its efficacy and side effects. ${ }^{21}$ It must be noted that in initiating opioids, regular repeat consultations are often needed to monitor for dose titration, adherence, aberrant use and adverse effects. ${ }^{22}$ Containment measures during the pandemic may pose difficulties in monitoring of opioid use. Face-to-face consultations may be postponed or cancelled, opioid prescriptions may not be filled in time due to stay home notices (SHN) or quarantine, and telemedicine has legal and safety limitations in monitoring opioid consumption. For patients who are already on long-term opioid therapy, there is a need to ensure that they continue to receive these medications to avoid withdrawal symptoms, and there should be regular follow-ups to gradually wean them to the lowest effective dose possible.

\section{Continuing Essential Pain Services}

\section{Cancer Pain Management}

Cancer patients may experience a considerable amount of pain as a result of the tumour pathology and cancer treatment. Management of cancer-related pain is an ethical responsibility of healthcare professionals to relieve unnecessary suffering and hence all treatment for cancer pain should be continued in spite of pandemic containment measures. ${ }^{23}$ The joint statement by ESRA and ASRA have also classified cancer-related pain procedures such as intrathecal pump insertions and refills as urgent pain procedures during the pandemic. ${ }^{1}$ Neurolytic procedures should also be considered to help increase the duration and efficacy of pain relief. ${ }^{24}$ Telemedicine should be considered for palliative care and cancer pain patients in view of their lowered immune system and increased susceptibility to infection. Even though clinic visits are limited, continued vigilance should be practised by both the pain and palliative care physicians, as well as the oncological teams to monitor disease progression and recurrence.

\section{Pain Management and the Elderly}

The incidence and prevalence of chronic pain increases with age, however pain can often be underreported in the geriatric population as many of the elderly patients may believe that pain is a normal process of aging. ${ }^{25}$ Safe distancing measures during the COVID-19 outbreak can be a particularly socially isolating period for them when household visits from families are restricted, hence the increasing the risk of worsening anxiety, depression, and functional decline from pain and immobility. Multidisciplinary involvement is paramount, and these should include efforts in collaborations with other specialties and community nursing teams, galvanising support from next of kins, easy access to help hotline numbers and ensuring that there is regular monitoring of patients who are at risk.

\section{Role of Primary Care}

Patients with stable pain conditions may be discharged to the care of their primary care physician with proper correspondences on their treatment regime. Primary care physicians have an important role to play in managing patients with chronic pain, working in tandem with pain specialists through monitoring of the pain conditions, providing medication refills when needed and aiding to reduce visits to emergency care units. At the start of COVID-19 in Singapore, many primary care clinics were converted to public health preparedness clinics (PHPCs) to consolidate the primary care clinic response to national emergencies. ${ }^{26}$ With the growing numbers of COVID-19 positive patients, more general practitioners (GP) are involved in the war against the virus, be it in PHPCs or in swab and community care facilities, and they inadvertently have less time to devote to pain 
management and other chronic illnesses. Coupled with the population's general reluctance to visit primary care clinics unless they are acutely unwell, chronic pain patients may not have as quick or often access to primary care for pain consultations or medication refills. Should the pandemic worsen, resources will be even more limited. Community collaborations have thus been put in place to optimise the role of primary care even during a lockdown situation, to provide accessibility to clinical care and support for the chronic pain patient.

\section{Community Collaborations for Chronic Pain Patients}

The deferment of specialist pain clinic consultations and limited access to primary care can increase morbidity and mortality. ${ }^{27-29}$ It is imperative to have a plan in place for the continued support of pain patients who may otherwise run out of essential pain management medications leading to worsening pain, functional decline and adverse psychological sequelae, prompting multiple emergency department (ED) attendances and untoward hospital admissions.

Collaboration is fundamental for pandemic preparedness with integration across health care sectors. ${ }^{30}$ In Singapore, coordination of care for our pain patients was done through collaboration with community healthcare teams, primary care physicians, hospital pharmacists and social service to provide for timely and effective responses to cater to the needs of the elderly and vulnerable, help reduce ED visits and hospital admissions. Palliative home-care teams supported terminal cancer patients in the community. In addition, community teams also provided education and guidance needed for patients and carers to participate in their own care.

Community team referrals were made on physician request for vulnerable patients who were elderly, had impaired mobility and/or multiple comorbidities, needing closer monitoring for pain and medication reconciliation. Categorisation of patients was done through telephone interviews and review of medical records. Stable patients were offered deferment of clinic appointments and home delivery of medication refills. Patients on regulatory authority-controlled drugs like opioids had medication refills via a strict verification protocol, with close monitoring of opioid effects. Pain clinic visits with appropriate screening for COVID-19 were continued for those who required consultation. Essential home or hospital consultations were restricted to a maximum of 30 minutes, with personal protection aids. Reviews by community teams and hospital teams were documented on a common electronic medical records portal for seamless access of information.

\section{Use of Telemedicine in Pain Management}

Telemedicine has been encouraged as an alternative to clinic consultations to comply with safe distancing and stay home measures. The use of telemedicine and appropriate triaging through telehealth were reported to be useful in managing pain services during COVID-19. ${ }^{31}$ At the time of writing, tele and video consultation platforms with enhanced security features are vigorously being set up and encouraging buy-ins from physicians and patients to reduce the number of physical clinic consults. Psychiatry and allied health teams can also make use of these platforms to conduct cognitive behavioural therapy sessions and exercise sessions for chronic pain patients respectively. Challenges in video consultations include poor access to and unfamiliarity with the technology especially in the elderly and less educated. Hence, there is a need for careful patient selection. The extremely infectious and insidious nature of the SARS-CoV-2 virus, however, means that this may become the new normal in view of a likely prolonged COVID-19 period. It is also important to realise that the medicolegal implications of telemedicine use are largely unknown. Like in all clinical disciplines, pain management assessment necessitates a detailed clinical examination to rule out any evolving diagnoses which will affect management strategies. At present, telemedicine is limited only to follow-up patients with stable pain conditions. New case consults, patients on opioid medications and patients with worsening pain conditions not responsive to treatment regime will still require face-to-face consults for detailed assessments.

\section{Conclusion}

This is a difficult period for all healthcare workers and patients. The chronic pain patient is especially vulnerable during a pandemic crisis and their needs are likely to be neglected or even deemed non-essential. However, it is important to realise that severe chronic pain is often debilitating and there is a need to ensure continuity of care for suffering patients through teleconsultations and community-based multidisciplinary care when physical face-to-face consults are not possible. At the same time there is a need to consider the risks and benefits of current pain management therapies, medications and interventions in the context of the COVID-19 pandemic - weighing the risks of potentially worsening outcomes should the pain patient contract COVID-19 and the benefit of pain improvement through the prescribed care plans. The authors hope that the above recommendations and experience will help to guide decision making in the care of the chronic pain patient. 


\section{REFERENCES}

1. American Society of Regional Anesthesia, Pain Medicine Recommendations on Chronic Pain Practice during the COVID-19 Pandemic [Internet]. Available from: https://www.asra.com/page/2903/ recommendations-on-chronic-pain-practice-during-the-covid-19pandemic. Accessed on 30 March 2020.

2. Russell CD, Millar JE, Baillie JK. Clinical evidence does not support corticosteroid treatment for 2019-nCoV lung injury. Lancet 2020;395:473-5.

3. Arabi YM, Mandourah Y, Al-Hameed F, Sindi AA, Almekhlafi GA, Hussein MA, et al. Corticosteroid Therapy for Critically Ill Patients with Middle East Respiratory Syndrome. Am J Respir Crit Care Med 2018;197:757-67.

4. Lee N, Chan AKC, Hui DS, Ng EKO, Wu A, Chiu RWK, et al. Effects of early corticosteroid treatment on plasma SARS-associated Coronavirus RNA concentrations in adult patients. J Clin Virol 2004;31:304-9.

5. Lee DTS, Wing YK, Leung HCM, Sung JJY, Ng YK, Yiu GC, et al. Factors associated with psychosis among patients with severe acute respiratory syndrome: a case-control study. Clin Infect Dis 2004;39:1247-9.

6. Xiao JZ, Ma L, Gao J, Yang ZJ, Xing XY, Zhao HC, et al. Glucocorticoid-induced diabetes in severe acute respiratory syndrome: the impact of high dosage and duration of methylprednisolone therapy. Zhonghua Nei Ke Za Zhi 2004;43:179-82.

7. Li YM, Wang SX, Gao HS, Wang JG, Wei CS, Chen LM, et al. [Factors of avascular necrosis of femoral head and osteoporosis in SARS patients' convalescence]. Zhonghua Yi Xue Za Zhi 2004;84:1348-53.

8. World Health Organization. Clinical management of severe acute respiratory infection when novel coronavirus (2019-nCoV) infection is suspected: interim guidance, 28 January 2020 [Internet]. World Health Organization; 2020. Report No.: WHO/nCoV/Clinical/2020.3. Available from: https://apps.who.int/iris/handle/10665/330893. Accessed on 01 April 2020

9. FPM-COVID-19-Steroid-Statement-2020.pdf. Available from: https:// fpm.ac.uk/sites/fpm/files/documents/2020-03/FPM-COVID-19Steroid-Statement-2020.pdf . Accessed on 30 March 2020.

10. Rosati R, Schneider BJ. Systemic Effects of Steroids Following Epidural Steroid Injections. Curr Phys Med Rehabil Rep 2019; 7:397-403.

11. Waljee AK, Rogers MAM, Lin P, Singal AG, Stein JD, Marks RM, et al. Short term use of oral corticosteroids and related harms among adults in the United States: population based cohort study. BMJ 2017;357:j1415.

12. Chutatape A, Menon M, Fook-Chong SMC, George JM. Metabolic and endocrinal effects of epidural glucocorticoid injections. Singapore Med J 2019;60:140-4.

13. RECOVERY Collaborative Group, Horby P, Lim WS, Emberson JR, Mafham M, Bell JL et al. Dexamethasone in Hospitalized Patients with Covid-19 - Preliminary Report. N Engl J Med 2020: NEJMoa2021436.

14. Nocom G, Ho KY, Perumal M. Interventional management of chronic pain. Ann Acad Med Singapore 2009;38:150-5.

15. Zhang H, Penninger JM, Li Y, Zhong N, Slutsky AS. Angiotensinconverting enzyme 2 (ACE2) as a SARS-CoV-2 receptor: molecular mechanisms and potential therapeutic target. Intensive Care Med 2020;46:586-90.

16. Voiriot G, Philippot Q, Elabbadi A, Elbim C, Chalumeau M, Fartoukh M. Risks Related to the Use of Non-Steroidal Anti-Inflammatory Drugs in Community-Acquired Pneumonia in Adult and Pediatric Patients. J Clin Med Res 2019;8:786.

17. Anti-inflammatoires non stérö̈diens (AINS) et complications infectieuses graves - Point d'Information - ANSM : Agence nationale de sécurité du médicament et des produits de santé [Internet]. Available from: https://ansm.sante.fr/S-informer/Points-d-information-Pointsd-information/Anti-inflammatoires-non-steroidiens-AINS-etcomplications-infectieuses-graves-Point-d-Information . Accessed on 01 April 2020.

18. Fu Y, Cheng Y, Wu Y. Understanding SARS-CoV-2-Mediated Inflammatory Responses: From Mechanisms to Potential Therapeutic Tools. Virol Sin 2020;35:266-271.

19. 19. Frank JW, Lovejoy TI, Becker WC, Morasco BJ, Koenig CJ, Hoffecker L, et al. Patient Outcomes in Dose Reduction or Discontinuation of Long-Term Opioid Therapy: A Systematic Review. Ann Intern Med 2017;167:181-91.

20. Sacerdote P. Opioid-induced immunosuppression. Curr Opin Support Palliat Care. 2008 Mar;2(1):14-8.

21. Budd K. Pain management: is opioid immunosuppression a clinical problem? Biomed Pharmacother 2006;60:310-7.

22. Ho KY, Chua NH, George JM, Yeo SN, Main NB, Choo CY, et al. Evidence-based guidelines on the use of opioids in chronic non-cancer pain--a consensus statement by the Pain Association of Singapore Task Force. Ann Acad Med Singapore 2013;42:138-52.

23. Weinstein SM, Janjan N. Management of Pain. 2015 Jun 1 [cited 2020 May 28]; Available from: https://www.cancernetwork.com/ cancer-management/management-pain . Accessed on 28 May 2020.

24. Kurita GP, Sjøgren P, Klepstad P, Mercadante S. Interventional Techniques to Management of Cancer-Related Pain: Clinical and Critical Aspects. Cancers 2019;11:443.

25. Kaye AD, Baluch A, Scott JT. Pain management in the elderly population: a review. Ochsner J 2010;10:179-87.

26. Hsu LY, Chia PY, Lim JF. The Novel Coronavirus (SARS-CoV-2) Epidemic. Ann Acad Med Singapore 2020;49:105-7.

27. Spiegel P, Sheik M, Gotway-Crawford C, Salama P. Health programmes and policies associated with decreased mortality in displaced people in postemergency phase camps: a retrospective study. Lancet 2002;360:1927-34.

28. Fernandez LS, Byard D, Lin C-C, Benson S, Barbera JA. Frail elderly as disaster victims: emergency management strategies. Prehosp Disaster Med 2002;17:67-74.

29. Supporting people with long-term conditions (LTCs) during national emergencies - CEBM [Internet]. CEBM. [cited 2020 Jun 3]. Available from: https://www.cebm.net/covid-19/supportingpeople-with-long-term-conditions-ltcs-during-national-emergencies/ Accessed on 3 June 2020.

30. Wynn A, Moore KM. Integration of primary health care and public health during a public health emergency. Am J Public Health 2012;102:e9-12.

31. Song X-J, Xiong D-L, Wang Z-Y, Yang D, Zhou L, Li R-C. Pain Management During the COVID-19 Pandemic in China: Lessons Learned. Pain Med 2020;21:1319-1323. 